\title{
$\Omega$
}

Andrea Aliverti

D

Dipartimento di Elettronica, Informazione e Bioingegneria Politecnico di Milano, Milan, Italy.

\section{The respiratory muscles during exercise}

\section{Physiology masterclass}

\section{How is the "ventilatory pump" made?}

From a functional point of view, there are three groups of respiratory muscles: the diaphragm, the rib cage muscles and the abdominal muscles. Each group acts on the chest wall and its compartments, i.e. the lung-apposed rib cage, the diaphragmapposed rib cage and the abdomen. Contraction of the diaphragm expands the abdomen and the lower part of the rib cage (abdominal rib cage). The rib cage muscles, including the intercostals, the parasternals, the scalene and the neck muscles, mostly act on the upper part of the rib cage (pulmonary rib cage) and are both inspiratory and expiratory. The abdominal muscles act on the abdomen and the abdominal rib cage and are expiratory. When each muscle group contracts alone or the contraction is predominant compared to the other groups, undesirable effects (such as "paradoxical" inward or outward motion during inspiration and expiration, respectively) occur on at least one of the compartments. A highly coordinated recruitment of two or three muscle groups is required to avoid these effects. During breathing at rest, this is accomplished by the coordinated activity of the diaphragm and inspiratory rib cage muscles. Normally no expiratory muscles are used [1].

\section{How does the ventilatory pump work during exercise?}

During exercise the increased ventilatory demands determine an increased neural drive to the respiratory muscles. This determines an increased mechanical power developed by the muscles. Muscle power is equal to velocity of shortening multiplied by pressure.

Differently than rest, during exercise the diaphragm is primarily a "flow generator". This means that its mechanical power is mainly expressed as velocity of shortening rather than pressure. Conversely, rib cage and abdominal muscles are primarily "pressure generators", i.e. develop the pressures required to move the rib cage and abdomen, respectively [1].

Differently than rest, during exercise the expiratory muscles play an active role in breathing. Within each single breath their action is highly coordinated with that of the inspiratory rib cage muscles. During inspiration, while the rib cage muscles contract, the abdominal muscles gradually relax, and vice versa during expiration. This mechanism has several effects: 1 ) it prevents rib cage distortion; 2) the diaphragm is unloaded and can act as a flow generator; and 3) the volume of the abdomen is decreased below resting levels
Cite as: Aliverti A. Physiology masterclass: The respiratory muscles during exercise. Breathe 2016; 12: 165-168. 
$[1,2]$. As a result, end-expiratory lung volume is decreased during exercise (figure 1) and the mechanics of breathing is optimised for several reasons. Tidal volume occurs in the most compliant part of the respiratory system; the diaphragm is lengthened and thus works near its optimal length; at each breath part of the required inspiratory work is previously stored in the form of elastic energy during the previous expiration [3].

\section{How do respiratory muscles undertake the increased ventilatory demands of exercise?}

At moderate levels of exercise, metabolic requirements increase in parallel with alveolar ventilation, arterial blood-gas tensions and acid-base balance are maintained close to their levels at rest. The mechanics of the breathing pattern is regulated so precisely that the work performed by the respiratory muscles is minimised.
At higher levels of exercise up to maximal exercise, the pressures produced by the respiratory muscles are well below their maximum. At maximal exercise, the oxygen consumed by the respiratory muscles to breathe is only $\sim 10 \%$ of the total [4]. However, this is only true for healthy subjects not those who are trained athletes. In fact, in highly fit endurance athletes the pressure produced by inspiratory muscles can approach the maximum and expiratory pressures are increased to levels at which dynamic compression of the airways determines expiratory flow limitation [5]. This phenomenon also frequently occurs in elderly subjects due to the age-related loss of lung elastic recoil [6], and is a common feature of patients with chronic obstructive pulmonary disease, not only during exercise but also at rest in the most severe cases. When expiratory flow is limited, end-expiratory lung volume has to be increased to allow for further increases of flow. In other words, expiratory flow limitation causes the so called "dynamic hyperinflation". At high operational lung volumes, the inspiratory muscles have to overcome a higher elastic load offered by the lung and chest wall [7]. Moreover, they are shorter and,

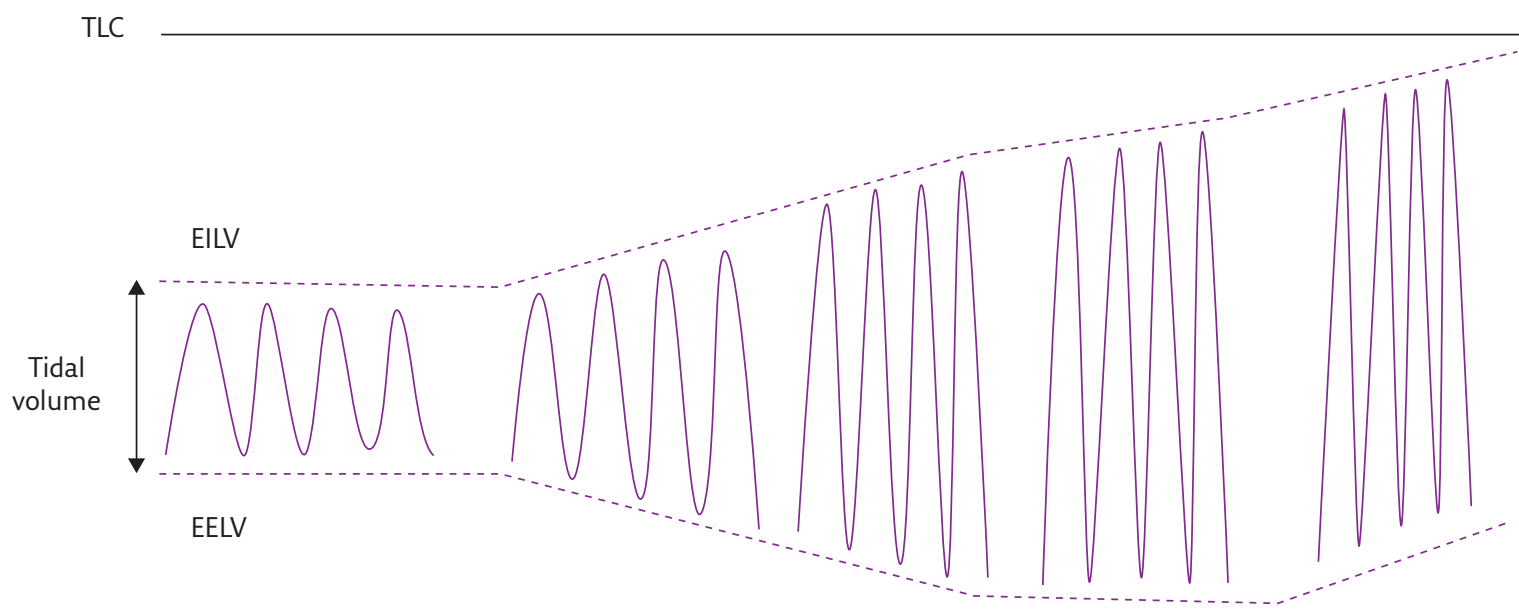

RV

Rest Light Moderate Hard Very hard Maximal

Diaphragm

Rib cage muscles

Abdominal muscles Not active

Features
Pressure generator (inspiration)

Pressure generators (inspiration)
Flow generator

Pressure generators (inspiration)

Pressure generators (expiration)

$\uparrow$ EILV

$\downarrow$ EELV

Positive circulatory effects of respiratory muscle contraction
Inspiration pressure approx. maximum

Expiration pressure approx. maximum

Expiratory flow limitation

$\uparrow$ EELV

$\uparrow$ blood flow $/ \mathrm{O}_{2}$ supply to respiratory muscles

Respiratory muscle fatigue

Metaboreflex

Negative circulatory effects of respiratory muscle contraction 
therefore, less capable of generating pressure. As a result, in these conditions the oxygen consumed by the respiratory muscles is increased $[4,8]$.

\section{Are respiratory muscles prone to fatigue?}

The high levels of respiratory muscle work that must be sustained throughout heavy exercise cause respiratory muscle fatigue. Fatigue of the respiratory muscles could initiate the metaboreflex, leading to vasoconstriction of limb locomotor muscle vasculature, exacerbating peripheral fatigue of working limb muscles and, via feedback, intensifying effort perceptions, thereby contributing to limitation of high-intensity endurance exercise performance [9]. However, whether the respiratory muscle metaboreflex is sufficient to prevail on the local vasodilator effects present in locomotor muscles and redistribute blood flow to respiratory muscles is still an open question.

\section{Is there a competition amongst muscles for the available oxygen and blood flow, and which muscle comes first?}

Skeletal muscle blood flow can range from 2 to $4 \mathrm{~L} \cdot \mathrm{kg}^{-1} \cdot \mathrm{min}^{-1}$ in humans. A similar, simultaneous demand from all muscles would require a cardiac output much greater than the highest never recorded. Therefore, there is competition for the limited, available cardiac output, a "pie" that has to be divided among all the skeletal muscles during whole body exercise. A further question is whether there is any hierarchy between respiratory and locomotor muscles and which limb muscle group receives a greater or smaller slice of the pie, of the total available cardiac output. It is clear that blood flow is distributed among the different limb muscles. In fact, the addition of arm exercise to leg exercise attenuates blood flow in the legs, while the addition of leg exercise to arm exercise reduces blood flow in the arms [10]. Conversely, it is not yet fully clear if respiratory muscles have a higher priority than locomotor muscles. Increasing or decreasing the work of breathing had the reciprocal effect on blood flow in the exercising legs, suggesting that the respiratory muscles demonstrate some sort of dominance over the locomotor muscles [11]. In trained cyclists, however, blood flow to the rib cage muscles (intercostals) is lower during exercise than when the same level of ventilation is maintained in the absence of limb movement, suggesting that blood flow is controlled in a similar way to other muscles with no evidence of priority over limb muscles [12]. It is likely that, as several animal studies suggest, blood flow to the diaphragm is less affected by sympathetic stimulation than other skeletal muscles; however, this is still to be confirmed. Certainly, less blood flow to the respiratory muscles promotes inadequate oxygen transport, and contributes to determining fatigue during heavy sustained exercise when arterial oxygen saturation can drop to $<85 \%$. In addition, reductions in limb blood flow and oxygen transport in response to fatiguing respiratory muscle work would be expected to impair limb locomotor muscle function [13]. Exercising in hypoxia exacerbates these effects and the increased work of breathing during hypoxia significantly contributes to both limb muscle fatigue and reduction in exercise tolerance.

\section{Does respiratory muscle contraction have any circulatory effect during exercise?}

The abdominal muscles, together with the diaphragm, might play a role as an "auxiliary heart" during exercise. In fact, at each act of breathing a significant amount of blood, presumably from the splanchnic vasculature, is shifted between the trunk and the extremities contributing to increase cardiac output $[14,15]$. To a presumably lesser extent, lower limb muscle contraction contributes to venous return facilitating the propulsion of blood from the skeletal muscle vasculature, through the so called "skeletal muscle pump". However, these mechanisms are only valid at moderate levels of exercise [16]. During heavy exercise, expiratory flow limitation and prolonged expiratory time result in higher average positive intrathoracic pressures that reduce ventricular transmural pressure and act like a Valsalva manoeuver, decreasing the rate of ventricular filling during diastole and reducing stroke volume, venous return and cardiac output. These effects of respiratory muscles on the cardiovascular system compromise systemic oxygen delivery [17] and make the limb muscles even more susceptible to fatigue.

\section{Are respiratory muscles coordinated with limb muscles?}

In several vertebrates running and breathing are "phase locked" so that the same number of steps occurs during each breath [18]. Although breathing and stepping frequencies are sometimes independent, tuning of locomotor and ventilatory muscles is often seen in humans during activities that involve impact loading with each foot strike, such as walking and running [19]. This reduces the energy cost of breathing, optimises the action of the muscles that contribute to both functions, allows for 
body stabilisation during motion, and utilises trunk bending and inertial movements of soft-tissues to augment inspiratory and expiratory flow by passively assisting the action of respiratory muscles, particularly the diaphragm because the abdominal viscera directly attach to this muscle [20].

\section{How to improve respiratory muscle performance during exercise}

Unloading the respiratory muscles during exercise by using low-density gas mixtures (such as heliox), mechanical ventilators or supplemental oxygen is neither practicable nor allowed for healthy athletes. What can be done in order to improve the fatigue resistance and mechanical efficiency of respiratory muscles is training. Although there is still no definitive evidence as to whether it is possible to improve exercise tolerance, reliable recent studies showed that respiratory muscle training has a small but probable and significant effect on endurance exercise performance. What needs to be determined is the mechanism or combination of mechanisms by which respiratory muscle training improves exercise performance: relief of respiratory muscle fatigue; relief of limb muscle fatigue; attenuation of the respiratory muscle metaboreflex; and relief of the discomfort associated with high levels of respiratory muscle work [21-23].

\section{Are there differences between men and women?}

Women have smaller lungs and airways than heightand age-matched men, and are also likely to develop expiratory flow limitation more often than men. For a given ventilation, women have a greater absolute oxygen cost of breathing and this represents a greater fraction of the total oxygen uptake compared to men. Although neither men nor women reach their maximal effective ventilation during exercise, women approach this value closer than men. Hence, the greater oxygen cost of breathing in women means that a greater fraction of total oxygen uptake and cardiac output is directed to the respiratory muscles, influencing exercise performance [24].

\section{Conflict of interest}

None declared.

\section{References}

1. Aliverti A, Cala SJ, Duranti R, et al. Human respiratory muscle actions and control during exercise. J Appl Physiol 1997; 83: 1256-1269.

2. Henke KG, Sharratt M, Pegelow D, et al. Regulation of endexpiratory lung volume during exercise. J Appl Physiol 1988; 64: 135-146.

3. Stubbing DG, Pengelly LD, Morse JL, et al. Pulmonary mechanics during exercise in normal males. J Appl Physio 1980; 49: 506-510

4. Aaron EA, Seow KC, Johnson BD, et al. Oxygen cost of exercise hyperpnea: implications for performance. J Appl Physiol 1992; 72: 1818-1825.

5. Aliverti A. Lung and chest wall mechanics during exercise: effects of expiratory flow limitation. Respir Physiol Neurobiol 2008; 163: 90-99.

6. Turner JM, Mead J, Wohl ME. Elasticity of human lungs in relation to age. J Appl Physiol 1968; 25: 664-671.

7. Agostoni E, Rahn H. Abdominal and thoracic pressures at different lung volumes. J Appl Physiol 1960; 15: 1087-1092.

8. Harms CA, Wetter TJ, McClaran SR, et al. Effects of respiratory muscle work on cardiac output and its distribution during maximal exercise. J Appl Physiol 1998; 85: 609-618.

9. Dempsey JA, Sheel AW, St Croix CM, et al. Respiratory influences on sympathetic vasomotor outflow in humans. Respir Physiol Neurobiol 2002; 130: 3-20.

10. Secher $\mathrm{NH}$, Volianitis S. Are the arms and legs in competition for cardiac output? Med Sci Sports Exerc 2006; 3: 1797-1803.

11. Harms CA, Babcock MA, McClaran SR, et al. Respiratory muscle work compromises leg blood flow during maximal exercise. J Appl Physiol 1997; 82: 1573-1583.

12. Vogiatzis I, Athanasopoulos D, Habazettl $H$, et al Intercostal muscle blood flow limitation in athletes during maximal exercise. J Physiol 2009; 587: 3665-3677.

13. Romer LM, Polkey MI. Exercise-induced respiratory muscle fatigue: implications for performance. J Appl Physiol 2008; 104: 879-888.
14. Uva B, Aliverti A, Bovio D, et al. The "abdominal circulatory pump": an auxiliary heart during exercise? Front Physiol 2016; 6: 411.

15. Aliverti A, Uva B, Laviola M, et al. Concomitant ventilatory and circulatory functions of the diaphragm and abdominal muscles. J Appl Physiol (1985) 2010; 109: 1432-1440.

16. Miller JD, Pegelow DF, Jacques AJ, et al. Skeletal muscle pump versus respiratory muscle pump: modulation of venous return from the locomotor limb in humans. J Physiol 2005; 563: 925-943.

17. Aliverti A, Dellaca RL, Lotti P, et al. Influence of expiratory flow-limitation during exercise on systemic oxygen delivery in humans. EurJ Appl Physiol 2005; 95: 229-242.

18. Boggs DF. Interactions between locomotion and ventilation in tetrapods. Comp Biochem Physiol A Mol Integr Physiol 2002; 133: 269-288.

19. Daley MA, Bramble DM, Carrier DR. Impact loading and locomotor-respiratory coordination significantly influence breathing dynamics in running humans. PLOS One 2013; 8: e70752.

20. Bramble DM, Carrier DR. Running and breathing in mammals. Science 1983; 219: 251-256.

21. Verges $S$, Lenherr $O$, Haner $A C$, et al. Increased fatigue resistance of respiratory muscles during exercise after respiratory muscle endurance training. Am J Physiol Regul Integr Comp Physiol 2007; 292: R1246-R1253.

22. Witt JD, Guenette JA, Rupert JL, et al. Inspiratory muscle training attenuates the human respiratory muscle metaboreflex. J Physiol 2007; 584: 1019-1028.

23. Sales AT, Fregonezi GA, Ramsook AH, et al. Respiratory muscle endurance after training in athletes and non-athletes: a systematic review and meta-analysis. Phys Ther Sport 2016; 17: 76-86.

24. Sheel AW, Dominelli PB, Molgat-Seon Y. Revisiting dysanapsis: sex-based differences in airways and the mechanics of breathing during exercise. Exp Physiol 2016; 101: 213-218. 